\title{
A Monte Carlo Model for the Simulation of the Electrodeposition of CoNi Alloys
} onto Glassy Carbon

\author{
A. C. Frank ${ }^{a}$, E. P. M. Leiva ${ }^{b}$, and P. T. A. Sumodjo ${ }^{a}$ \\ ${ }^{a}$ Instituto de Química, Universidade de São Paulo, São Paulo - SP, Brasil \\ ${ }^{\mathrm{b}}$ Unidad de Matemática y Física, Facultad de Ciencias Químicas, Universidad Nacional \\ de Córdoba, Córdoba, Argentina
}

In this work, a Monte Carlo method for the simulation of the early stages of CoNi electrodeposition is presented. The model was constructed based on previous experimental parameters obtained with $\mathrm{Co}, \mathrm{Ni}$ and $\mathrm{CoNi}$ electrodeposition onto a glassy carbon electrode. The core of the proposed simulation model consists of two Monte Carlo modules. The first one is a lattice grand-canonical Monte Carlo module which intends to calculate the energies of all possible events associated with metal deposition, namely adsorption, desorption and surface diffusion. The second one is a Kinetic Monte Carlo module which associates the previously calculated energies with reaction rates and probabilities of occurrence of the events, being analogous to the temporal evolution of the metallic electrodeposition.

\section{Introduction}

It is known that the physical properties and morphology of an electrodeposit strongly depend on several parameters of the electrodeposition process, such as solution composition, $\mathrm{pH}$, temperature, potential and current density (1). Therefore, electrodeposition requires an adequate control of the electrochemical phase formation process and a deep understanding of the reaction mechanism to produce an electrodeposit with desired properties. Particularly, the first stages of the electrodeposition play a very important role on the subsequent formation of the bulk metallic phase. So, it is very important for one to have an adequate control and knowledge of the first stages of electrodeposition to obtain a deposit with the desired physical and structural properties.

At the moment, theoretical and experimental electrochemists are working hand-inhand towards a deeper understanding of the early stages of the electrodeposition process. With regard the experimental approach, over the past decades, improvements in in situ nanoscopy techniques have made it possible to observe the temporal evolution of the surface morphology in electrodeposition experiments. This progress in experimental techniques has spurred theoretical investigations into the properties of nanostructures. From a theoretical approach, a large variety of models are generally used to describe nucleation and crystal growth. However, many of the related problems, particularly those concerning the growth or decay of structures, are difficult to treat by proper theory. Therefore, they have become the domain of computer simulations. But it was only in the last few years, due to the recent and fast advance of computational technology, that simulations of these processes gained more attention and helped scientists to understand more profoundly the metallic nucleation and growth phenomena and avoid trial and error experimentation.

Up to now many different approaches are taken depending on the problem at hand. In general, the methods can be divided in three groups: deterministic, quasi-deterministic and stochastic. The first one refers to the classical mechanics method called Molecular Dynamics; the second one refers to a variety of methods as Brownian dynamics and 
Langevin dynamics and the later, a purely stochastic method that enters in the classification of Monte Carlo techniques. There are a lot of Molecular Dynamics simulations performed for crystal growth such as vapor deposition, growth from melts and growth from supersaturated solutions, but the conventional Molecular Dynamics method cannot be used for the reactions of electrodeposition due to the large timescale of these processes compared to the small timescale that a Molecular Dynamics method can simulate. For that reason and due to the stochastic nature of the electrodeposition process, the Monte Carlo method has become a useful tool to study crystal growth.

There are various works in the development of Monte Carlo models, or hybrid models based in part in Monte Carlo models, to simulate the electrodeposition of a variety of systems. The majority of the works concerns copper electrodeposition, due to the applications of copper in the microelectronics industry as the material of choice for on-chip interconnects (2-5). Beside $\mathrm{Cu}$, other systems have been studied, like the electrodeposition of silver, gold or platinum (6-8). There are many others different systems have been modeled, implicating in many different Monte Carlo models constructed for each specific situation. However, the Co and its alloys have not received much attention up to now. Specifically, although the focus of many experimental studies, due to their good magnetic and mechanical properties (9), the electrodeposition of CoNi alloy has not yet been studied by computer simulations.

In this context, the present work tries to contribute to the comprehension of some essential characteristics of the CoNi deposition by means of a very simple model. In this paper the description of the model is reported. The results will be published in a forthcoming paper. The model was constructed based in part on previous experimental data obtained with $\mathrm{Co}, \mathrm{Ni}$ and $\mathrm{CoNi}$ electrodeposition onto a glassy carbon electrode. The core of the proposed simulation model consists of two Monte Carlo modules. The first one is a lattice-gas grandcanonical Monte Carlo module and intends to calculate the energies of all possible events associated with metal deposition, namely adsorption, desorption and surface diffusion. The second one is a Kinetic Monte Carlo module, which associates the previously calculated energies with reaction rates that are proportional to the probabilities of occurrence of the events. This module aims to simulate the temporal evolution of the metallic electrodeposition.

\section{The Model}

\section{$\underline{\text { General considerations }}$}

The Monte Carlo (MC) method is based on the assumption that all mathematical problems can be tackled by a probabilistic analogous that can be solved by stochastic sampling experiments. All possible system configurations are sampled according to a previously defined probability density function. From the states generated in a random walk through the configurations, one can calculate the average of the different properties that characterize the system. The probability of occurrence of one configuration $p_{i}$ can be given, for example, by:

$$
p_{i}=\frac{\exp \left[-U\left(r_{i}\right) / k_{b} T\right]}{\int \exp \left[^{-U\left(r_{i}\right)} / k_{b} T\right] d r_{i}}
$$

where $\boldsymbol{r}_{i}$ refers to the spatial coordinates of the $i$ th atom, $k_{b}$ is the Boltzmann constant, $T$ is the temperature and $U\left(\boldsymbol{r}_{i}\right)$ is the potential energy associated with the $\boldsymbol{r}_{i}$ configuration. The integral in this equation runs over all the configurations of the system and constitutes the 
spatial term of the partition function. By this method, the average value of a property of the system can be calculated by taking an average of its value over the generated configurations.

However, to determine the probability of a given configuration, it would be necessary to know the odds of occurrence of all configurations, which is not always feasible. In the case of continuous variables, an integration of probability density over the entire space of configurations would be necessary. To tackle these often computationally prohibitive difficulties of the MC method, we applied the Metropolis algorithm (10). This is one of the most popular and efficient important sampling methods to perform an MC simulation, since it is based on computing the ratio of the probabilities of two consecutive configurations, making it unnecessary to determine the probability of the other configurations in all iterations.

Furthermore, to reduce the computational cost of MC simulations using the Metropolis algorithm, a lattice model was chosen rather than a continuous model (off lattice model) (11). Lattice models for computer simulations are of widespread use in studies of adsorption on surfaces because they allow dealing with a large number of particles at a lower computational cost when compared to continuous models. To use this kind of approximation, it is important to verify if there is a crystallographic misfit present in the studied system. If the crystallographic misfit between the involved atoms is negligible, it is a good approximation to assume that the adatoms adsorb on defined discrete sites on the surface, given by the positions of the substrate atoms. In order words, it is important to see if the lattice spacings for the substrate and for the deposited metal are significantly different. Here we have adopted the values of $2.4624 \AA$ for the carbon lattice spacing (12), $2.2590 \AA$ for Co and $2.3766 \AA$ for $\mathrm{Ni}$ (13). The resulting crystallographic misfits are 8.26 for Co and 3.48 for Ni. Following the criteria proposed by Rojas (14) that a lattice model can be used if the crystallographic misfit is lower than 8.3 , we can adopt a lattice model in this study.

Square lattices with periodical boundary conditions are used in the present work to represent the surface of the electrode. Each lattice point represents an adsorption site for an adsorbate or a substrate atom. The former may adsorb or desorb on each randomly selected site, while the latter are frozen. An occupation number is defined (that is, 0 for an unoccupied site, 1 for a site occupied by an substrate type atom, 2 or 3 for a site occupied by a adsorbate type atom, depending if it is $\mathrm{Co}$ or $\mathrm{Ni}$ ).

Finally, another consideration that deserves mention concerns the kind of ensembles used. In the grand-canonical ensemble, used in this study, besides the volume $V$ and temperature $T$, the chemical potential $\mu$ is fixed, but the number of particles $N$ may vary. This ensemble is particularly useful in Electrochemistry in many cases, such as chronoamperometry and cyclic voltammetry, since the control of electric potential difference involves the control of $\mu$ of some species in the electrochemical interface. The value of the electrical potential of the working electrode is related to $\mu$ by:

$$
V=\left(E_{c}-\mu\right) / z
$$

where $E_{c}$ is the cohesion energy of the species present at the electrochemical interface and $z$ is its charge.

In this point, some important questions arise in the construction of the model. The first deals with the modeling of the surface of glassy carbon used in the lattice models, simulating the working electrode. The second concerns the probability density function in a system containing $\mathrm{Co}, \mathrm{Ni}$ or both, and a glassy carbon substrate. To achieve this, it is essential the previous determination of the metal-metal and metal-substrate interatomic potentials in the form of analytic functions. This leads to the third point: once the potential functions are found, the energies of all possible configurations should be stored in an energy table in order to use appropriately the advantages of choosing a lattice model. 


\section{Glassy carbon surface}

Nowadays, the structure of the glassy carbon is widely discussed in the literature, but there is no consensus yet due to the complexity of the spatial arrangement of the carbon atoms. However, as is inevitable in a computational modeling program, some approximations must be made. In this work, the surface of glassy carbon was considered as a hexagon arrangement of carbon atoms, like the graphite surface. This is a valid approach, because in both the hybridization of carbon atoms is $\mathrm{sp}^{2}$ and there are studies in the literature (15) that relate the structure of glassy carbon with graphite or fullerene, with the spatial arrangement of carbon atoms in the form of hexagons.

Therefore, the glassy carbon electrode surface was approximated as a hexagonal close-packed ( $h c p$ ) surface. All the calculations were done for the $h c p$ (111) lattice, although on real glassy carbon surfaces both $f c c$ and $h c p$ domains are present due to the surface reconstruction. Four available adsorption sites for metal adsorption were scrutinized and symbolized with the color Xs in the Figure 1, depending on how many carbon atoms are bonding the adsorbate atom. Each configuration has a different energy and should be computed through an analytical function in order to be incorporated into the $\mathrm{MC}$ program to simulate the metal deposition on glassy carbon.

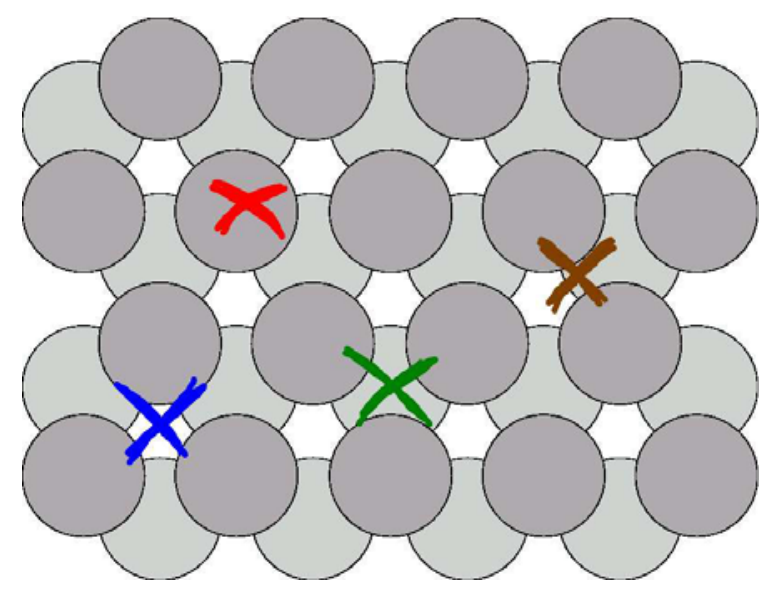

Figure 1. Representation of the four possible configurations of the adsorption sites on the hcp surface of glassy carbon. The upper $\mathrm{X}$ represents an adsorption site directly above a carbon atom, with only one carbon-metal bond. The $\mathrm{X}$ on the far right represents a adsorption site located between 2 carbon atoms. The left $\mathrm{X}$ in the bottom line symbolizes the active site in which the adsorbate interacts with 3 carbon atoms. The right $\mathrm{X}$ in the bottom line stands for an active site with 4 binding carbon atoms, one of them located at the second monolayer of the substrate.

However, in a real system there are not only perfect flat monolayers of substrate, but actually an imperfect surface, with kinks and steps. Thus, the surface energy of the active sites may be different from one another, as it occurs in a real electrode due to its roughness. A metal atom of adsorbate can therefore adsorb preferentially at one site over another. To incorporate this variable in the program, we have simulated some surface defects most commonly observed in experiments, as is shown in Figure 2. The 0D clusters correspond to the adsorption of isolated metal atoms, the adsorption on kink sites or on vacancies of the first lattice plane of the substrate. The $1 \mathrm{D}$ phases are related to the adsorption at the border of 
steps or monoatomic terraces and the 2D phases are related to the formation of monolayers. The $2 \mathrm{D}$ phases are the flat terraces. The different types of surface defects were created using the simulated annealing technique (16).

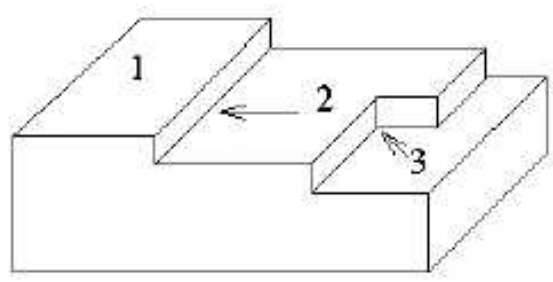

a

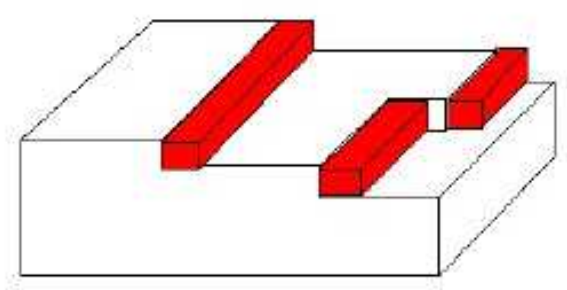

c

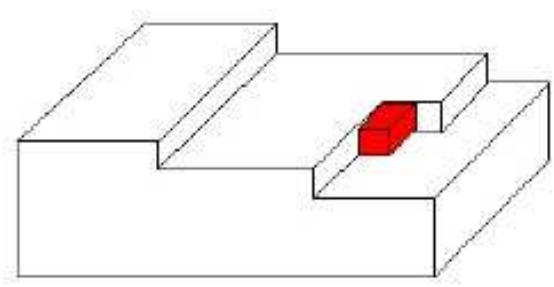

b

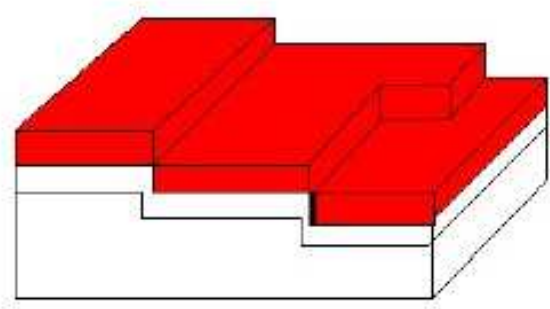

d

Figure 2. (a) The three types of active sites on the simulated surface of glassy carbon: (1) flat terraces, (2) steps and (3) kinks. b) First stage of metal deposition, on the kinks. c) Second stage, on the steps. d) Third stage, on the terraces. Extracted from (16).

\section{$\underline{\text { Metal-metal and metal-substrate interatomic potentials }}$}

The next step is to define the interaction between the atoms and the surface. A very important factor that should be taken into account in order to do proper modeling of a system is the quality of the interatomic potential used. The study of a system that includes a large number of atoms requires a model that is at the same time accurate and computationally simple. Here, we use classical many-body potential functions between carbon atoms and Co or Ni atoms, as well as among the transition metal atoms. This function is constructed by fitting binding energies from the Density Functional Theory (DFT) calculations made by Shibuta and Maruyama (13). A classic many-body potential was developed in the form of an analytical function of potential energy that depends on the number of metal-metal or metalcarbon atoms bonded. In DFT calculations, the binding energy is derived from the difference between the energies of the clusters $\mathrm{MC}_{\mathrm{n}}$ and $\mathrm{M}_{\mathrm{n}}(\mathrm{M}=\mathrm{Co}$ or $\mathrm{Ni}, \mathrm{n}=1-4)$ and the energies of the individual atoms. The binding energy from DFT calculations are fitted to the following functions:

$$
\begin{gathered}
E=V_{R}-V_{A} \\
V_{R}=\frac{D_{e}}{S-1} \exp \left[-\beta \sqrt{2 S}\left(r-R_{e}\right)\right] \\
V_{A}=B^{*} \frac{D_{e} S}{S-1} \exp \left[-\beta \sqrt{2 / S}\left(r-R_{e}\right)\right] \\
B^{*}=\left[1+b\left(N^{C}-1\right)\right]^{\delta}
\end{gathered}
$$


In these functions, $V_{R}$ and $V_{A}$ are Morse type repulsive and attractive terms, respectively, whereas $D_{e}$ and $R_{e}$ are the binding energy and equilibrium bond length, respectively. Potential parameters $S, \beta, b$ and $\delta$ determine the shape of the potential functions. For metal-carbon many-body potential functions, the many body effect is expressed as a function of carbon coordination number of a metal atom $N^{C}$. For metal-metal interactions, the $D_{e}$ and $R_{e}$ are expressed as direct functions of the metal coordination number $N^{M}$ instead of using the additional term $B^{*}$. It is noteworthy that $N^{C}$ and $N^{M}$ vary depending on the number of atoms bonded in the $\mathrm{MC}_{\mathrm{n}}$ and $\mathrm{M}_{\mathrm{n}}$ clusters and on the bond distances. More details can be found here (13). The final values of the parameters obtained by fitting are summarized in Table 1.

TABLE I. Potential parameters for metal-carbon and metal-metal interactions, obtained by fitting the many-body potential functions to the binding energies from the DFT calculations (13).

\begin{tabular}{ccccccc}
\hline & $\boldsymbol{S}$ & $\boldsymbol{\beta ( 1 / \AA )}$ & $\boldsymbol{D}_{\boldsymbol{e}}(\mathbf{e V})$ & $\boldsymbol{R}_{\boldsymbol{e}}(\AA)$ & $\boldsymbol{b}$ & $\boldsymbol{\delta}$ \\
\hline Ni-C & 1.3 & 1.8706 & 2.4673 & 1.7628 & 0.0688 & -0.6256 \\
Co-C & 1.3 & 1.3513 & 3.7507 & 1.6978 & 0.0889 & -0.5351 \\
Ni-Ni & 1.3 & 1.5700 & 1.4361 & 2.3766 & - & - \\
Co-Co & 1.3 & 1.5552 & 1.5704 & 2.2590 & - & - \\
Co-Ni & 1.3 & 1.5626 & 1.5032 & 2.3178 & - & - \\
\hline
\end{tabular}

As can be inferred from the larger $D_{e}$ value provided in Table 1, the bonding between $\mathrm{Co}$ and $\mathrm{C}$ is stronger than between $\mathrm{Ni}$ and $\mathrm{C}$. As a consequence, the equilibrium bond length of Co-C is lower than the Ni-C one. There is not much difference in the values of the metalmetal parameters when changing from $\mathrm{Co}$ to $\mathrm{Ni}$. The many-body interactions were taken into account for the $\mathrm{MC}_{\mathrm{n}}$ and $\mathrm{M}_{\mathrm{n}}$ clusters $(\mathrm{M}=\mathrm{Co}$ or $\mathrm{Ni}$ and $\mathrm{n}=1-4)$, in the configurations showed in the Figure 1. On calculating the energies of the deposition process using the fitted functions, it was verified that the minimum energy was obtained when a metal atom adsorbs onto a green active site depicted in Figure 1, which is the only that allows the adsorbate to be bonded with four carbons. Therefore, the lattice grand-canonical Monte Carlo assumes in the calculations that these are the only sites where deposition can take place.

\section{Energy Tables}

One of the main advantages of the lattice model is its simplicity, since it fixes the distances between the adsorption nodes, thus reducing the energy values of the system to a discrete set. Moreover, the potentials used are short ranged, a simplifying assumption to obtain the energy differences. The point is to consider the adsorption (or desorption) of a particle at a node immersed in a certain environment surrounding it. If pairwise additive interaction between nearest neighbors is assumed and interactions between more distant atoms are neglected, the thermodynamic properties of the system can be obtained from the thermodynamics of the Ising model for the lattice gas. Lattice gas models allow dealing with a relatively large number of particles at a relatively low computational cost.

Figure 3 shows the nearest neighbors active sites considered for the calculations of the adsorption energies of the metal atoms on the glassy carbon surface. There are the first neighbors (dark squares), the second neighbors (medium dark squares) and the third neighbors (light squares). Those adsorption sites located out of the cutoff radii of active site 13 are not considered to influence the energy of the process. Therefore, there are many possibilities of configurations, depending on what is the neighborhood of the active site considered. Each configuration has a specific energy associated with it, and all these energies 
are stored in an energy table, that will be used by the Monte Carlo method to calculate the probabilities of the possible moves the system may perform.

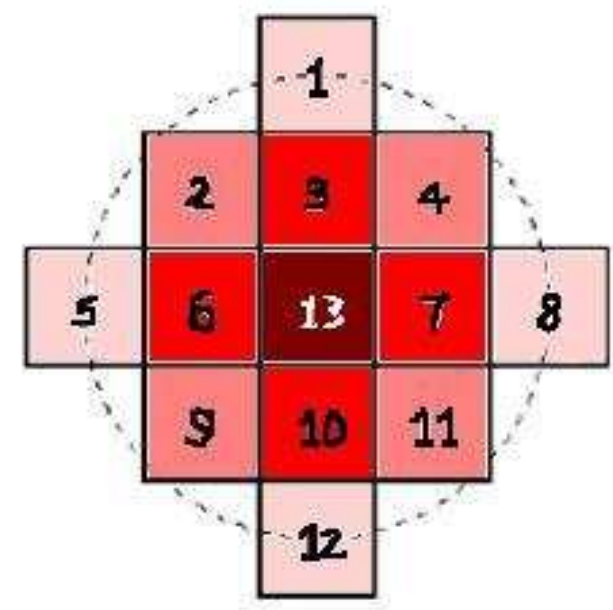

Figure 3. Nearest neighbors active sites considered for the calculations of the adsorption energies of the metal atoms on the glassy carbon surface.

\section{Grand-canonical Monte Carlo module}

The main objective of this module is, based on the energy tables of the system, to simulate the final configurations of the metal monolayer electrodeposited onto a defective glassy carbon surface after the application of various overpotentials. This is a way to know the final appearance of metal film formed, the degree of coverage of the surface and the preferred active sites for deposition.

From experimental data, it was verified that CoNi electrodeposition onto glassy carbon is an overpotential deposition (opd) process, which implies that the adsorption of the adsorbate on the foreign substrate is less favorable than that predicted by the Nernst equation. That was taken into account in our model, as the applied overpotential was an important variable of the computational program.

The grand-canonical MC module consists of three possible processes after the random choice of an adsorption site:

a) If the site is empty (occupation 0 ), the creation of an adsorbate atom is attempted (yielding occupation 2 or 3 depending if it is Co or $\mathrm{Ni}$ ): $\Delta N=+1$

(b) If the site is occupied by an adsorbate atom (occupation 2 or 3 depending if it is Co or $\mathrm{Ni}$ ), its desorption is attempted (occupation 0): $\Delta N=-1$

(c) If the site is occupied by a substrate atom (occupation 1) a surface diffusion process is tried: $\Delta N=0$.

Items (a) and (b) can understood as a kind of MC event that try to change the occupation number of a randomly selected active site. The glassy carbon surface contains a total of $M$ active sites and one of them is selected with probability $1 / M$. Since each adsorption site can have a total of 4 different occupations (empty - 0 , with $\mathrm{C}$ atom - 1 , Co atom - 2 and $\mathrm{Ni}$ atom - 3), this kind of MC step tries to change the occupation number of that site as follows:

$$
\operatorname{matrix}(i, j)=2(\text { or } 3)-\operatorname{matrix}(i, j)
$$


where matrix $(i, j)$ contains the occupation number of the site $(i, j)$. Thus, if the original occupation was 0 , it means that the site was empty and there is an attempt to put a particle of adsorbate there (occupation 2 or 3). The number of particles in the system will change by $\Delta N$ $=+1$ and the energy change of the system will be $\Delta E=E_{a d s}$ (where $E_{a d s}$ is the energy of a particle entering the site $(i, j)$, depending on the neighborhood of the active site). If the occupation number of the selected site is 2 or 3 , meaning that there is a particle of adsorbate there, there is a try to remove it (yielding an occupation 0). In this case, the number of particles of the system will change by $\Delta N=-1$ and the energy change of the system will be $\Delta E=-E_{a d s}$. In all cases, the proposed events will be either accepted or rejected according to a probability that depends on the energy change and on the applied overpotential:

$$
p=\min \left[1, \exp \left(-\frac{\Delta E-\mu \Delta N}{k_{b} T}\right)\right]
$$

A diffusion event of type (c) can only occurs if the occupation number of the active site selected is 1 . To simulate the diffusion of metal particles on the surface, they are numbered from 1 to $N$, regardless of where they are located. One adsorbate particle is random selected and the program tries to move it to a nearby site, with probability $1 / 12$ each according to Figure 4 . If the final site is occupied, the metal particle stays where it was. If the site is free, a move to this location is tried, according to a probability:

$$
p=\min \left[1, \exp \left(-\frac{\Delta E}{k_{b} T}\right)\right]
$$

where $\Delta E=E_{\text {ads }}^{\text {final }}-E_{\text {ads }}^{\text {initial }}$, the difference between the energy of the final and the initial configuration of the system.

The total energy of the system at each MC step was calculated in advance and stored in the energy table, which is accessed by the grand-canonical MC module to calculate the probabilities given in the equations [8] and [9]. During this stage of the grand-canonical MC simulation, the final (equilibrium) configuration of the system can be compared by varying the applied overpotential, the initial surface and the presence of defects, for example. These equilibrium configurations of the deposited film can be also compared with experimental data obtained by means of various microscopy experiments.

\section{$\underline{\text { Kinetic Monte Carlo module }}$}

The second MC module constructed in this work is the Kinetic Monte Carlo module (KMC), which is described in this section. The grand-canonical MC method proposed is very useful to calculate the equilibrium properties of the system, but does not deliver any information about the kinetics of the nucleation and growth processes. The KMC module is used to fill that gap. When the dynamics of the physical system can be described as a thermally activated process, such as adsorption, desorption and diffusion, it is possible to construct a KMC algorithm that allows the simulated system to evolve according to the physical system. Furthermore, the theoretical basis for the KMC method is defined in terms of Poisson processes, which are stochastic processes in which events occur continuously and independently of one another. Those criteria are all met here in this work, so a KMC method is suitable for study the present problem.

Unlike the grand-canonical MC module, in the KMC the Metropolis algorithm cannot be used because it does not met the kinetic criteria in the sense that a lower energy final state is not always favorable in detriment to a higher energy initial state, because there is an activation barrier that the system need to surpass in order to reach the final state. So, the transition state theory is used as an algorithm to select the acceptance criteria for each transition. The key point of the KMC method is that the rate of each process that may occur on the surface can be described by an Arrhenius type equation: 


$$
k=A \exp \left[\frac{E_{a}}{k_{b} T}\right]
$$

where $k$ is the experimental rate constant for the reaction, $A$ is the pre-exponential factor (or frequency factor) and $E_{a}$ is the Arrhenius activation energy. It is a common approximation to think of each of the processes considered in terms of a system oscillating around a minimum in the potential energy surface and making $A$ attempts to overcome the energy barrier per unit time. The probability of success depends on $T$ and the height of energy barrier, which is considered the activation energy of the process and is calculated from the binding energy $D_{e}$ of the DFT data.

Once all the processes that can occur in a certain step of the simulation are identified, each one will be assigned with a reaction rate $v_{i}$ inversely proportional to its activation energy (data obtained from the table of energies previously determined by the grandcanonical MC and calculated from the difference between the energies of the two processes). The probability of the process to occur can be represented in a straight line by a segment proportional to $v_{i}$, as is outlined in Figure 4 for a system in which only three processes can occur at a time $t$.

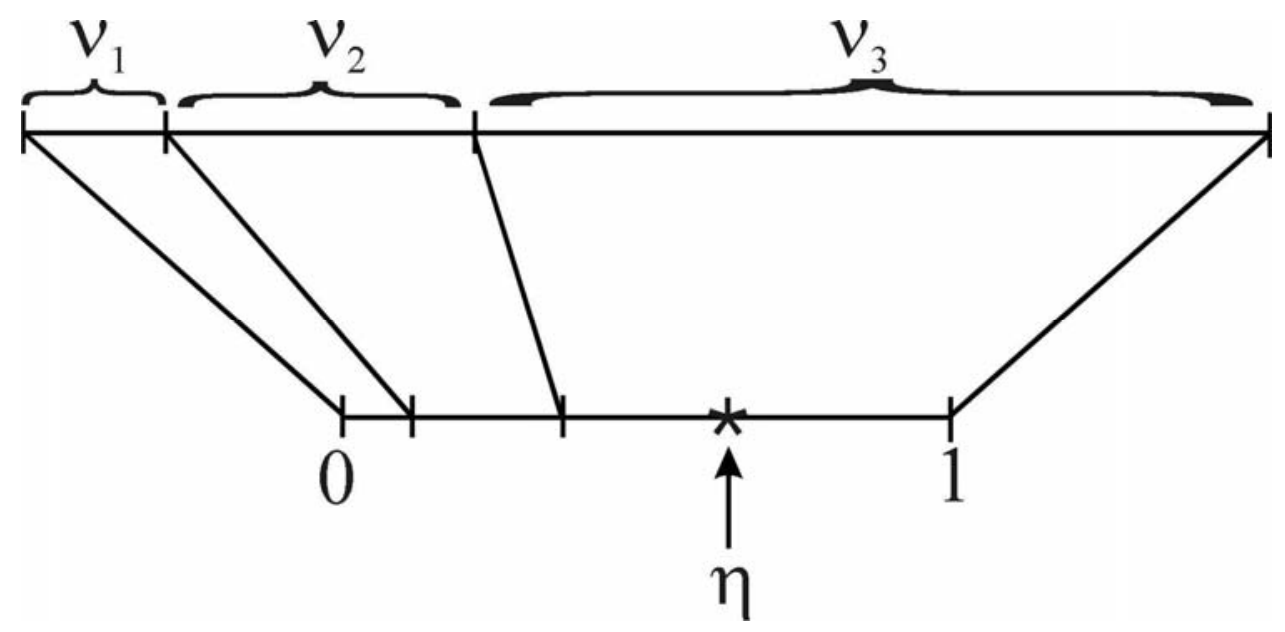

Figure 4. Illustration of the selection of a process in a Kinetic Monte Carlo simulation in which 3 processes may occur. The probability of occurrence of each process is symbolized by a segment of a straight line proportional to its rate $v_{i}$. The sum of all segments is normalized to a unitary vector. A process is selected by generating a random number $\eta$ between 0 and 1, and then the process corresponding to the segment on which the random number is found to fall is selected. Here $\eta$ falls in the segment corresponding to the process with rate $v_{3}$.

If the sum of all segments is normalized to unit length, the occurrence of a process can be selected from the generation of a random number uniformly distributed in the range 0 to 1. As shown in Figure 4, the process selected is that corresponding to the segment in which the random number fell. Since the probability of changing the current state of the system has a constant rate, the elapsed time $\Delta t$ until the change is accepted has an exponential distribution. Thus, $\Delta t$ is calculated as

$$
\Delta t=-\frac{\ln (\eta)}{\sum_{i} v_{i}}
$$

where $\eta$ is a random number uniformly distributed on $(0,1)$ and the sum index runs over all possible processes at a given time step. After letting the time advance by a random amount 
$\Delta t$ (which is completely independent of what was the random selected process), the configuration of the system is updated, a new set of rates $\left\{v_{i}\right\}$ is calculated, a new process is randomly chosen again and so on.

The algorithm for implementation of the KMC method used in this work can be layed out as follows, where $t$ indicates the time elapsed since the beginning of the simulation, $\mu$ indicates the random event to occur, $\mathrm{p}(\mu)$ is its probability, $\tau$ is the time that has elapsed between one event and another, and $\mathrm{P}(\tau, \mu)$ is the joint probability that the next event that occurs is associated with the index $\mu$ and the time elapsed between the last event and the next is $\tau$.

TABLE II. Steps of the Kinetic Monte Carlo algorithm proposed.

$* t=0$

* Define the initial state of the system

Step $0 *$ Calculate the rates associated to each one of the possible processes, based on the energy tables previously calculated.

* Define the sample times: $t_{1}<t_{2} t_{1}<t_{2}<\ldots<t_{\text {stop }}$

* Generate a pair of random numbers $(\tau, \mu)$, accordingly to the probability

Step 1 density function $P(\tau, \mu)$

* Update the time: $t=t+\tau$

* Make the changes associated with the $\mu$ event

Step $2 *$ Recalculate the rates associated with each event, taking into account the new state of the system

* If $t$ is greater than the sample time $t$, show the present state of the system

Step $3 *$ If $t>t_{\text {stop }}$, or if $p(\mu)=0$ for every $\mu$, the simulation ends. Otherwise, back to step 1

\section{Conclusions}

In this paper it was described the construction of a simulation model of the CoNi electrodeposition onto a glassy carbon electrode. It was divided into two modules using the MC method, each with its specifications and goals. The results of the simulations using this model will be published in a forthcoming paper.

Initially, the correct modeling of the glassy carbon surface and the determination of the metal-metal and metal-carbon interaction potential have been met. With these data, the first modules of the MC, the grand-canonical MC, was outlined to calculate the energy tables of all possible movements involved in electrodeposition of those metals on glassy carbon. It was considered the influence of the first, second and third neighbors in the adsorption energy of an atom in an environment containing different types of substrate defects. Having the energy tables, the probabilities of occurrence of deposition (adsorption), dissolution (desorption) and diffusion have been defined.

The other MC module used in this work was the KMC one. This model associates the activation energy of each possible movement in the system with an inversely proportional reaction rate constant. Each event then has a probability of occurrence and the system will be updated as new settings are being obtained. Using the KMC method, it can be obtained an analogous to the time evolution of the electrode surface during the CoNi electrodeposition process, from which can be theoretically determined the kinetic parameters and the nucleation and growth mechanisms. 


\section{Acknowledgments}

Financial support in the form of a research grant from FAPESP (Fundação de Amparo à Pesquisa do Estado de São Paulo) and a fellowship from CNPq (Conselho Nacional de Desenvolvimento Científico e Tecnológico) are gratefully acknowledged.

\section{References}

1. W. Schwarzacher and D. S. Lashmore, Trans. on Magn., 32, 3133 (1996).

2. T. O. Drews, R. D. Braatz and R. C. Alkire, J. Electrochem. Soc., 150 (11), C807 (2003).

3. T. O. Drews, A. Radisic, J. Erlebacher, R. D. Braatz, P. C. Searson and R. C. Alkire, J. Electrochem. Soc., 153 (6), C434 (2006).

4. A. Saedi, J. Electroanal. Chem., 588, 267 (2006).

5. L. Guo, A. Radisic and P. Searson, J. Phys. Chem. B, 109, 24008 (2005).

6. M. M. Mariscal, E. P. M. Leiva, K. Pötting and W. Schmickler, Appl. Phys. A, 87, 385 (2007).

7. M. M. Mariscal, S. A. Dassie and E. P. M. Leiva, J. Chem. Phys., 123, 184505 (2005).

8. O. A. Oviedo, C. E. Mayer, G. Staikov, W. J. Lorenz and E. P. M. Leiva, Surface Science, 600, 4475 (2006).

9. N. V. Myung, D. -Y. Park, B. -Y. Yoo and P. T. A. Sumodjo, J. Magn. Magn. Mat., 265, 189 (2003).

10. N. Metropolis, A. W. Rosenbluth, M. N. Rosenbluth, A. H. Teller and E. Teller, J. Chem. Phys., 21 (6), 1087 (1953).

11. M. M. Mariscal, E. P. M. Leiva, Cap. 2: Computer Simulations of Electrochemical Low-dimensional Metal Phase Formation. in: Electrocrystallization in Nanotechnology (1 ${ }^{\mathrm{a}}$ ed.), Wiley-VCH, Weinheim (Alemanha) (2006).

12. G. Chiarotti, Landolt-Börnstein - Group III Condensed Matter, 24c, 21 (1995).

13. Y. Shibuta and S. Maruyama, Comp. Mat. Sci., 39, 842 (2007).

14. M. I. Rojas, Surface Science, 569, 76 (2004).

15. P. J. F. Harris, Philosophical Magazine, 84 (29), 3159 (2004).

16. M. C. Giménez, M. G. Del Pópolo, E. P. M. Leiva, Electrochim. Acta, 45, 699 (1999). 